\title{
Direct Tests of a Pixelated Microchannel Plate as the Active Element of a Shower Maximum Detector
}

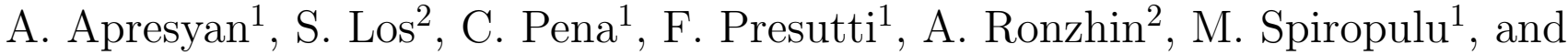 \\ S. $\mathrm{Xie}^{1}$ \\ ${ }^{1}$ California Institute of Technology, Pasadena, CA, USA \\ ${ }^{2}$ Fermi National Accelerator Laboratory, Batavia, IL, USA
}

\begin{abstract}
One possibility to make a fast and radiation resistant shower maximum detector is to use a secondary emitter as an active element. We report our studies of microchannel plate photomultipliers (MCPs) as the active element of a shower-maximum detector. We present test beam results obtained using Photonis XP85011 to detect secondary particles of an electromagnetic shower. We focus on the use of the multiple pixels on the Photonis MCP in order to find a transverse twodimensional shower distribution. A spatial resolution of $0.8 \mathrm{~mm}$ was obtained with an $8 \mathrm{GeV}$ electron beam. A method for measuring the arrival time resolution for electromagnetic showers is presented, and we show that time resolution better than 40 ps can be achieved.
\end{abstract}

\section{Introduction}

In order to collect large datasets needed for precise characterization of the Higgs boson, and in order to increase new physics discovery potential, future high-energy hadron colliders, as well as the High Luminosity LHC (HL-LHC) upgrades are expected to deliver peak luminosities in excess of $5 \times 10^{34} \mathrm{~cm}^{-2} \mathrm{~s}^{-1}$. With the increased instantaneous luminosity the simultaneous interactions per bunch crossing (pileup) increase the likelihood of confusion in the reconstruction of particles from the hard scatter interaction with those produced in different pileup interactions. The ability to discriminate jets, photons, and electrons produced in the events of interests from pileup becomes significantly degraded.

One way to mitigate the pileup confusion effects, complementary to precision tracking methods, is to perform a time of arrival measurement associated with a particular layer of the calorimeter, allowing for a time assignment for charged particles and photons. Achieving such a time measurement with resolution better than 20-30 ps can effectively reduce the impact of pileup by a factor of 5. The ultimate goal is to develop an electromagnetic calorimeter with the same capabilities as the ones used in the CMS and ATLAS experiments, but with the enhanced capability to achieve time measurements at precisions of $20-30$ ps. In this paper we continue our investigation of the development of such a calorimeter [1-4].

The use of micro-channel plates (MCP) as the active element of a shower-maximum detector or a calorimeter has been studied in the past [5, 6]. These studies demonstrated the linearity in the multiplicity of secondary shower particles that have energies that the MCP can detect. Such detectors are also a promising option for achieving time measurement precision at the level of a few tens of picoseconds [2, 4, 7]. Moreover, such devices are intrinsically radiation hard and thus would tolerate the harsh radiation environment at future hadron colliders, particularly when operated 
without the reliance on a photocathode. In reference [4, we have demonstrated that the intrinsic fluctuations of electromagnetic showers induce jitter on the time measurement that is less than $10 \mathrm{ps,}$ removing one important potential fundamental limitation. A further advantage of MCP's is their capability for highly segmented readout, allowing for the possibility of a highly granular calorimeter with sub-millimeter spatial resolution. Such high-granularity calorimeters have been studied in the context of detector concepts for the ILC [8] and the HL-LHC upgrade of the CMS experiment [9], indicating that such calorimeters have promising potential for substantial improvement in physics reach at the $\mathrm{TeV}$ scale. In this paper, we complement our past results [1-4] with additional studies of the position and time resolution for a calorimeter prototype with highly granular readout.

In this and the three previous papers we used three different MCP-PMTs:

- Photek 240: our most performant device, which provides the best time resolution, and excellent uniformity across the detector. The main parameters of the Photek 240 were reported in Ref. [2]. The pore size is $10 \mu \mathrm{m}$ and the distance from the photocathode to the first amplification stage is $5.3 \mathrm{~mm}$. The Photek 240 has a $41 \mathrm{~mm}^{2}$ circular sensitive area, and it was operated $4.8 \mathrm{kV}$ high voltage (HV). The gain at this voltage is about $10^{6}$. The non-uniformity of the time response of the signal is limited to below 3.9 ps across the full sensitive area.

- Photonis XP85011: the anode of this MCP is composed of 64 pads, arranged as an $8 \times 8$ matrix. The size of each pad is $6 \times 6 \mathrm{~mm}^{2}$. The pore size is $25 \mu \mathrm{m}$. The non-uniformity of the time response across the photocathode is 37 ps [2, 4]. The HV applied to the Photonis $\mathrm{XP} 85011$ was $2.4 \mathrm{kV}$, with a corresponding gain of $10^{6}$.

- Photonis XP85012: mostly identical to XP85011, also composed of 64 pixels arranged as an $8 \times 8$ matrix. Additionally it can be operated in a mode with a reverse voltage applied to photocathode, which enables us to effectively turn off any signals from the photocathode. When operated in this mode, the only signals are directly from secondary shower particles [3].

In this paper, we report on our studies of the high-granularity shower-maximum detector prototype that uses the Photonis XP85011 MCP as the active element. As demonstrated in reference [2], due to the fact that the input window is very thin, the signal in this device is dominated by direct detection of secondary shower particles, while Cherenkov photon signals contribute only $30 \%$ of the amplitude. The MCP is used to sample the electromagnetic shower induced by a beam of electrons impacting a tungsten absorber layer that has a thickness of about 4 radiation lengths $\left(X_{0}\right)$. The MCP-PMT is read out with a pixelated anode, with square pixels of size $6 \times 6 \mathrm{~mm}^{2}$. The energy of the electromagnetic showers is reconstructed using the total collected charge and the positions are reconstructed using a simple energy-weighting algorithm, described in Section 4. Through the use of a high-precision motorized stage, a position scan is performed during beam-tests and the position resolution of the shower-maximum detector is obtained. Finally, we investigate the precision of measuring the arrival time of electromagnetic showers with such a pixellated shower-maximum detector.

The paper is organized as follows. In Section 2 we describe the experimental setup used to perform the measurements, in Section 3 we present the event selection and pulse reconstruction, in Section 4 and 5 the results on measured position and timing resolutions are presented.

\section{Experimental Setup}

The experiment was performed at the MTEST location of the Fermilab Test Beam Facility using an $8 \mathrm{GeV}$ beam primarily comprised of electrons. A differential Cherenkov counter, located further upstream of the MTEST location, was used to enhance the purity of electrons and to suppress pions, by requiring a signal consistent with a passage of electrons through the device. All other detectors were placed inside a dark box lined with copper foil for electromagnetic shielding. A photograph of the experimental setup within the dark box is shown in Figure 1. A scintillator of size $1.7 \mathrm{~mm} \times 2.0 \mathrm{~mm}$ optically coupled to two photomultiplier tubes, one on each side, was 
used to trigger the data acquisition and to constrain the trajectory of the electrons from the beam. Downstream of the trigger, a tungsten absorber with a thickness of about $1 \mathrm{~cm}$, equivalent to about 4 radiation lengths, was placed. The Photonis XP85011 MCP-PMT with pixelated readout was set on a high precision motorized stage and placed behind the tungsten absorber. The precision of the motorized stage is about $0.1 \mathrm{~mm}$. To avoid unintended early showers due to interactions with the material of the casing and MCP device, the Photek 240 MCP-PMT was placed behind the Photonis XP85011 MCP-PMT.

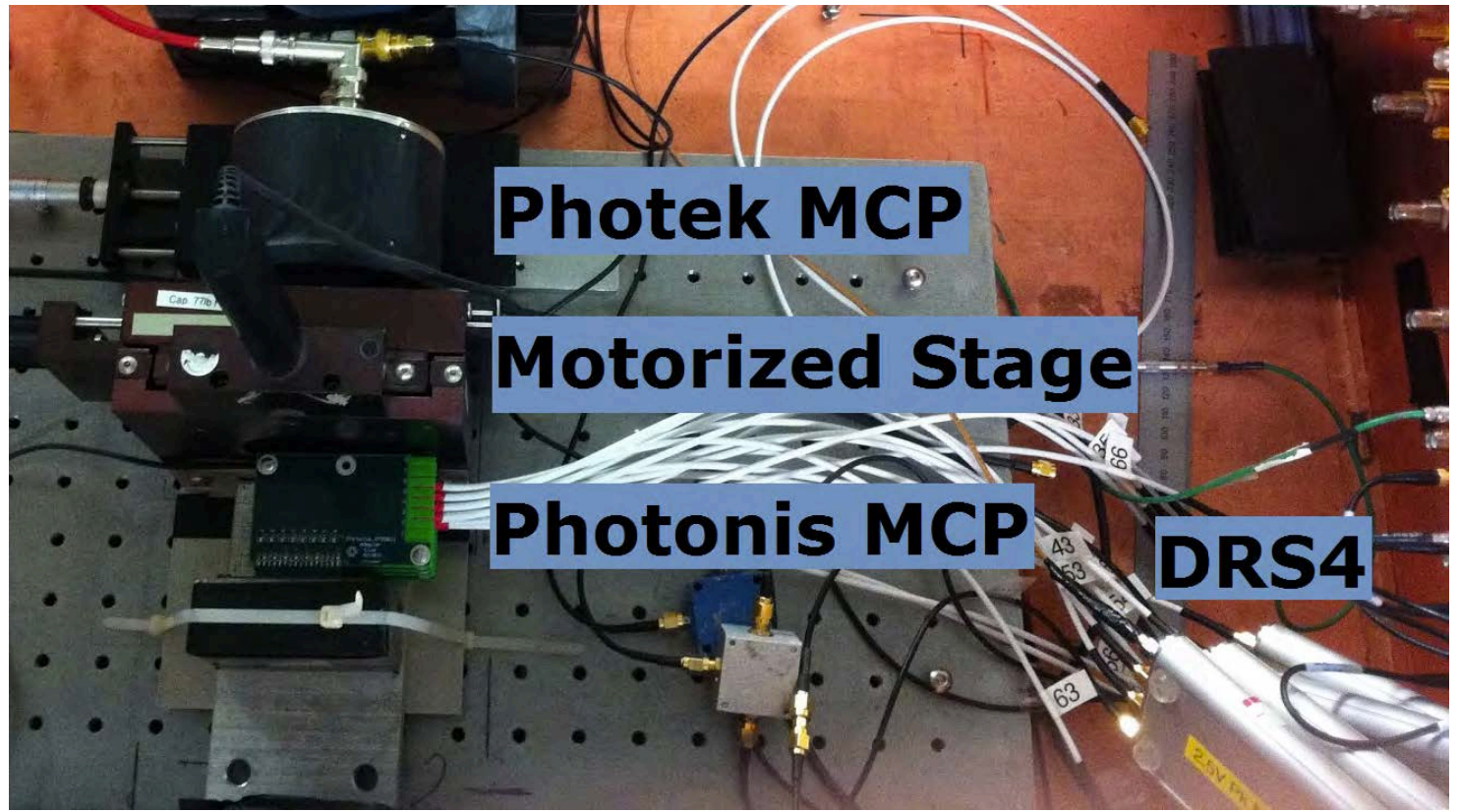

Figure 1: The experimental setup inside of the dark box is shown. The beam direction is from the bottom of the photograph to the top. The detector elements shown in the order from upstream to downstream of the beam are: the tungsten absorber, the Photonis XP85011 MCP-PMT located on the motorized stage, and the Photek 240 MCP-PMT used as a time reference detector. The DRS4 waveform digitizers are also shown on the lower right side.

An external view of the Photonis XP85011 MCP-PMT is shown on the left of Figure 2, and a schematic diagram is shown on the right. There are a total of 64 pixels arranged in an $8 \times 8$ square that can be read out individually. For our experiment, the nine pixels shown within the red square are used. During the course of the experiment we found that the pixel labelled 44 in Figure 2 did not function properly and was therefore not used in the analysis of the data.

Four DRS4 high speed waveform digitizers were used to acquire the signals from the Photek 240 MCP-PMT, the cherenkov counter, and the eight operational channels from the Photonis XP85011 MCP-PMT. In order to allow a synchronized readout of four separate DRS4 units we split the signals from the Photek 240 MCP-PMT into four, and connected them to each of the four DRS4 units, thus achieving a "calibration" between the four different units.

\section{Event Selection and Pulse Reconstruction}

Reconstruction of the signal pulses and timestamps is performed using the identical methods described in our past studies [1 3]. In Figure 3, we show example pulses from one pixel channel of the Photonis XP85011 MCP-PMT and the Photek 240 MCP-PMT digitized by the DRS4.

We measure time resolution as the standard deviation of the Gaussian fit to the time-of-flight distribution $t_{0}-t_{1}$, where $t_{0}$ is the time recorded at the "start" detector, and $t_{1}$ is that of the "stop" detector. To assign a time stamp for each signal pulse, we first determine the time position 


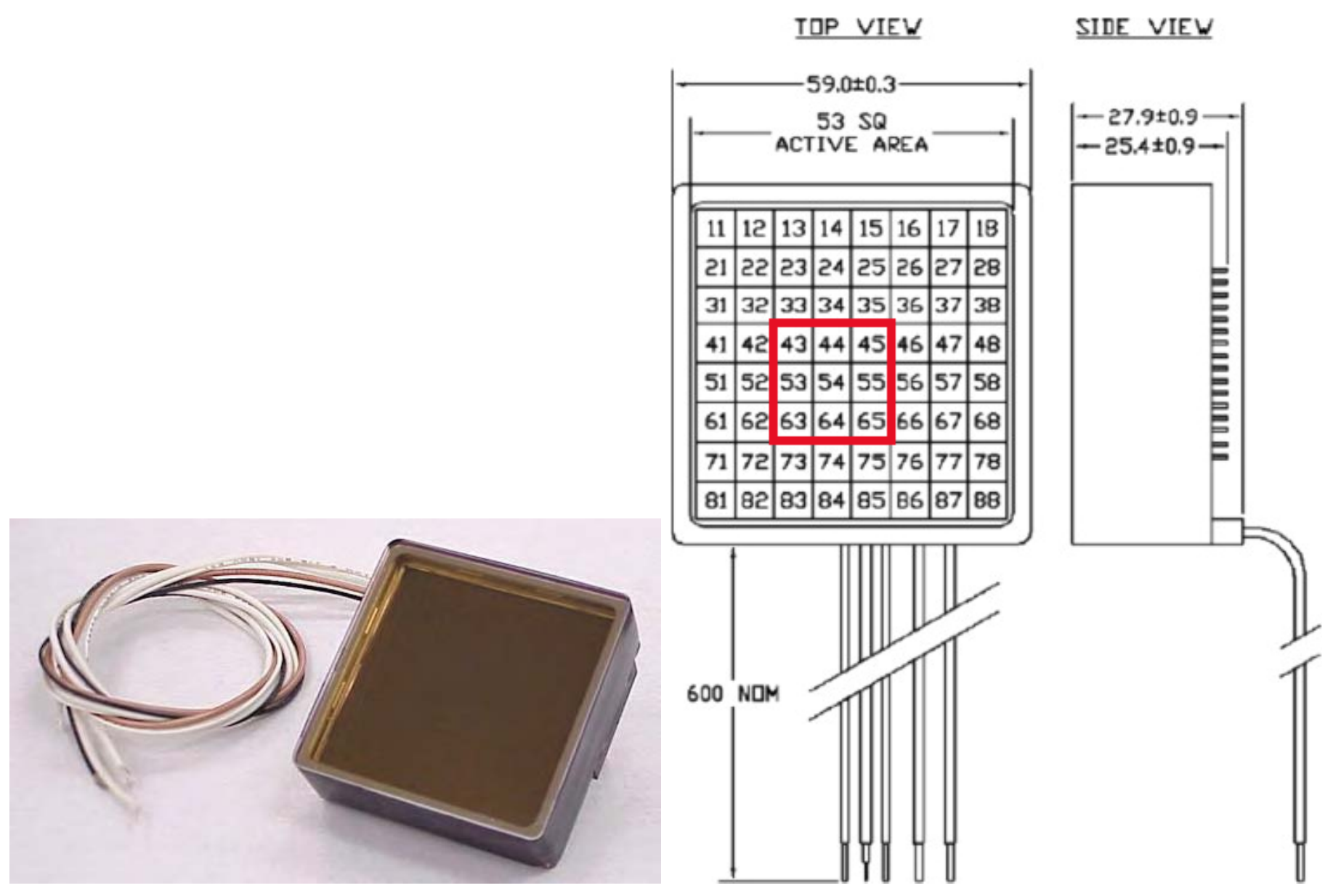

Figure 2: The external view of the Photonis XP85011 MCP-PMT is shown on the left, and the schematic diagram is shown on the right. The red square indicates the pixels used for the experiment and data analysis. 
of the pulse peak. A Gaussian function is fitted to the pulse maximum using three points before the maximum of the pulse peak and four points after the maximum. The mean value of the Gaussian was used as the time stamp for each pulse. A Photek 240 MCP-PMT, whose time resolution was previously measured to be less than 10 ps [3] was used as a "start" signal, while pulses from individual pixels on the Photonis XP85011 MCP-PMT were used as "stop" signals. More details on the pulse reconstruction algorithms that we use are presented in Ref. [2]. The integrated charge for each pulse is used as a proxy for the measured energy deposit in each channel, and is computed using four time samples before and after the peak of the pulse. Each time sample is approximately $0.2 \mathrm{~ns}$ in time. Events containing pulses above $500 \mathrm{mV}$ in amplitude are rejected as they saturate the DRS4. Only pulses with amplitude larger than $20 \mathrm{mV}$ are used for time measurements, to reduce the impact of the electronics noise in the DRS4. Other event selection and pulse cleaning procedures are used to eliminate abnormal pulses in the readout, as described in [2].
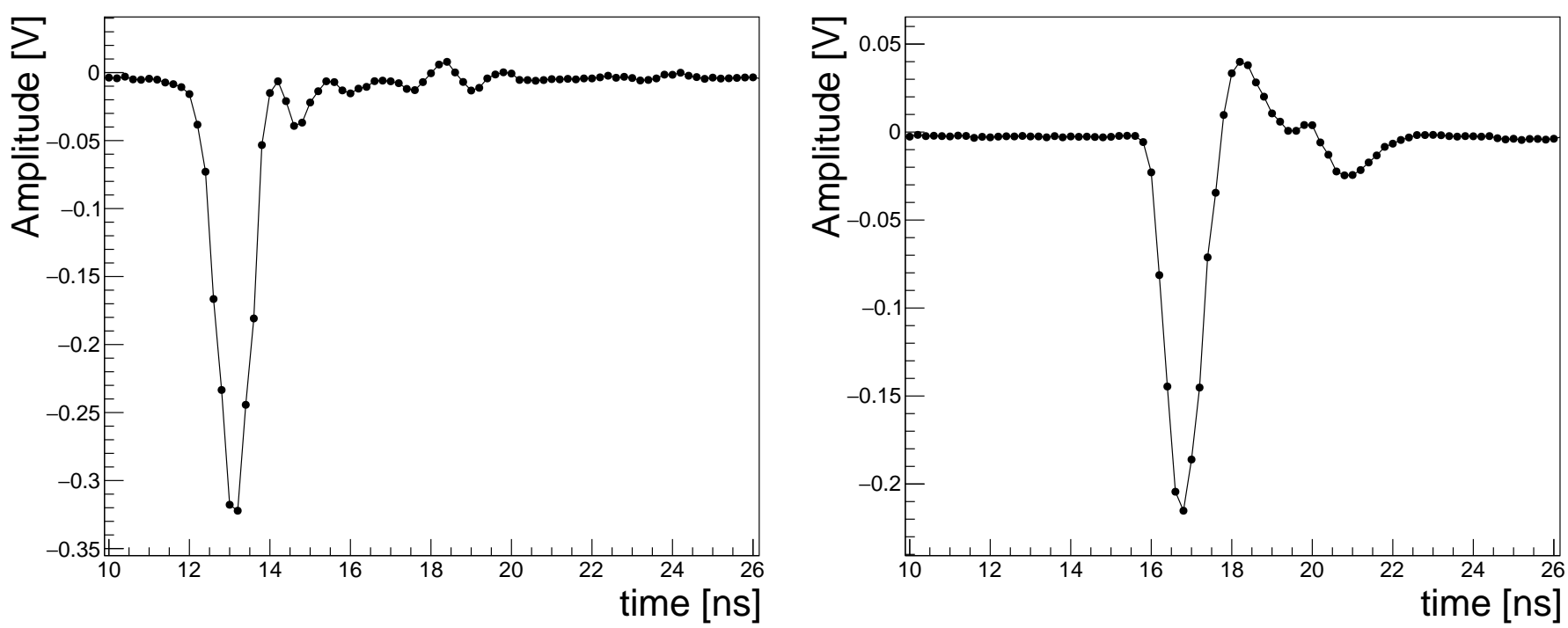

Figure 3: Example of a digitized signal from a single Photonis pixel (left) and Photek (right) MCP-PMT following a high-energy electron shower, via DRS4.

\section{Electromagnetic Shower Position Reconstruction and Resolution}

The transverse shape of electromagnetic showers is very well known and has a characteristic width given by the Moliere radius. For tungsten, the Moliere radius is about $9 \mathrm{~mm}$ and therefore we expect the shower to be contained within two of the pixels in the Photonis XP85011 MCP-PMT. In Figure 4, we show the mean charge measured in each of the pixels for one example run where the Photonis MCP-PMT was held in a fixed location approximately centered on the beam. The electron beam has a width of about $1 \mathrm{~cm}$.

Each electron impacting the shower-maximum detector will induce an electromagnetic shower, and we define such an occurrence as an event. For each event, we reconstruct the center position, $\vec{p}$ of the electromagnetic shower based on the the pixel positions weighted by the corresponding integrated charge as follows:

$$
\overrightarrow{\mathbf{p}}=\frac{\sum_{i \in \text { pixels }} Q_{i} \vec{p}_{i}}{\sum_{i \in \text { pixels }} Q_{i}}
$$

where $i$ labels the individual pixels, $Q_{i}$ is the charge collected in pixel $i$, and $\vec{p}_{i}$ is the vector 
Mean Charge Distribution

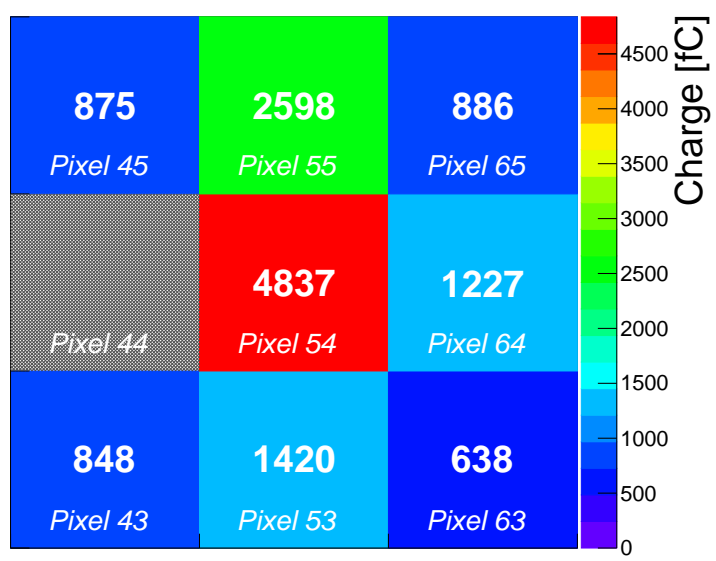

Figure 4: The mean charge measured for each pixel for one example run is shown. During this run, the Photonis MCP-PMT was held in the same location. Based on the distribution of the mean charge among the pixels, we can infer that the beam center is located in the upper half of the center pixel. Pixel 44 is not shown as it was found to be not operational.

describing the $x$ and $y$ coordinates of the center of pixel $i$. The origin of the coordinate system is chosen to be at the lower left corner of the $3 \times 3$ array of pixels.

Multiple runs were taken scanning different beam positions relative to the Photonis MCP-PMT by moving the motorized stage. In Figure 5 we show the distributions of the reconstructed shower positions for three example runs in which the beam was located near the top, center, and bottom of the center pixel. The distributions of the reconstructed $y$ coordinate for the three corresponding runs are shown together in Figure 6. The measured beam-spot is observed to move consistent with the known movement of the motorized stage.

For each run, we determine the center of the beam-spot by fitting the measured $x$ and $y$ coordinates with a gaussian function. The data from all runs are combined by considering the measured $x$ and $y$ coordinates relative to the center of the beam-spot (see Fig 7). We model the distribution of measured coordinates as a convolution of a flat distribution with width equal to the measured dimensions of the scintillator trigger and a gaussian resolution function. A maximum likelihood fit is performed on the data using this model, and the position resolution of the detector is measured as the width of the gaussian resolution function. We measure the position resolution as $0.55 \pm 0.2 \mathrm{~mm}$ in $x$-coordinate, and $0.91 \pm 0.01 \mathrm{~mm}$ in $y$-coordinates.

\section{$5 \quad$ Electromagnetic Shower Time Resolution}

The timestamps for each event for individual pixels of the Photonis MCP-PMT are reconstructed as described in Section 3. We reconstruct the timestamp of the entire electromagnetic shower using the same energy weighting procedure that was used above for the shower position reconstruction:

$$
t=\frac{\sum_{i \in \text { pixels }} Q_{i} t_{i}}{\sum_{i \in \text { pixels } Q_{i}}}
$$

where $i$ labels the individual pixels, $Q_{i}$ is the charge collected in pixel $i$, and $t_{i}$ is the reconstructed time-stamp for pixel $i$. Alternatively, we also study the time resolution using the single pixel with the highest energy deposit measurement. In Figure 8 we show the time distributions for these two methods of shower time reconstruction.

In Figure 9, we compare the time resolution for electromagnetic showers measured using the two methods described above. The time resolution for the pixel with the largest energy deposit is 

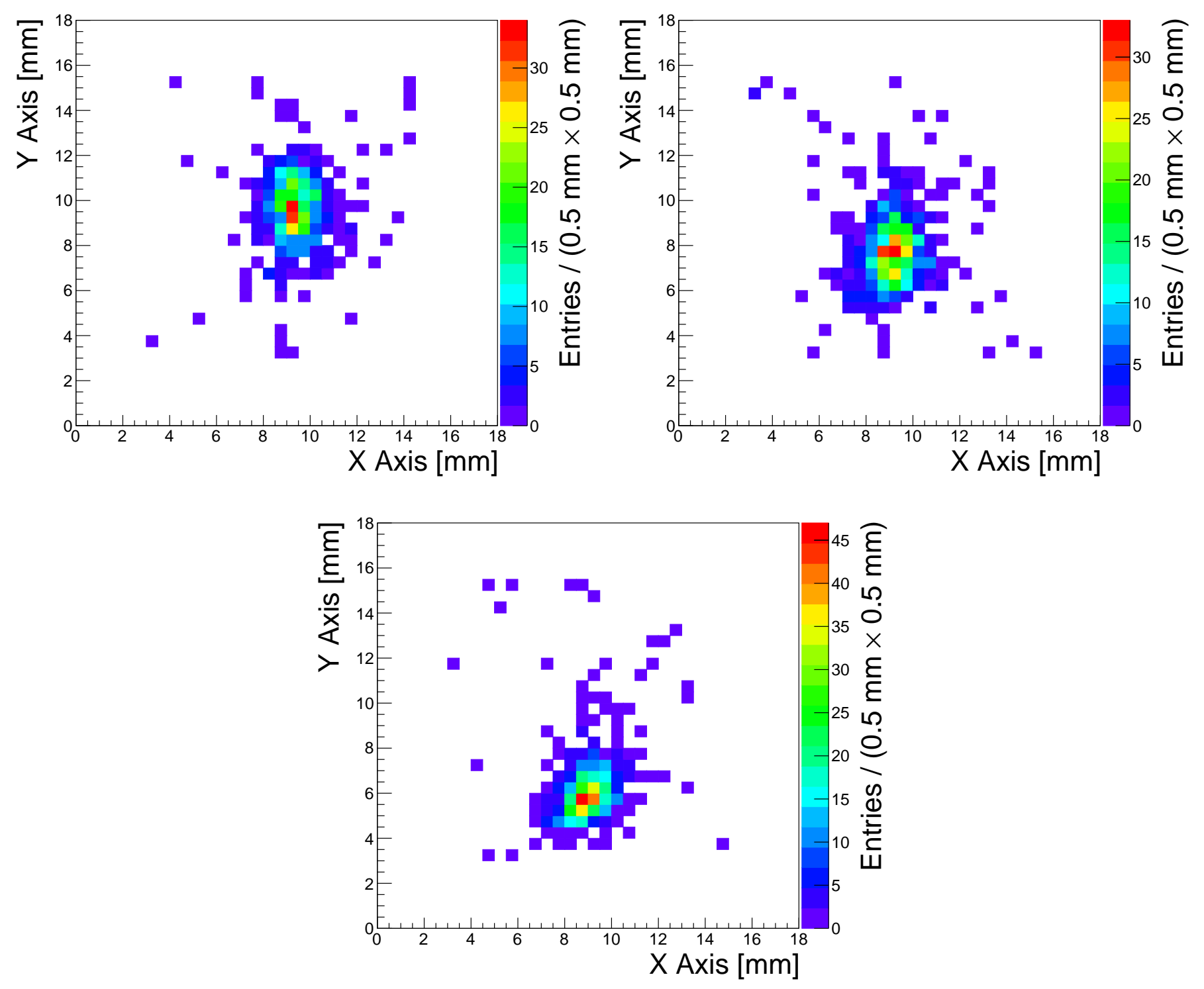

Figure 5: The distribution of reconstructed shower positions is shown for three runs with the beam centered near the top, center, and bottom of the central pixel. 


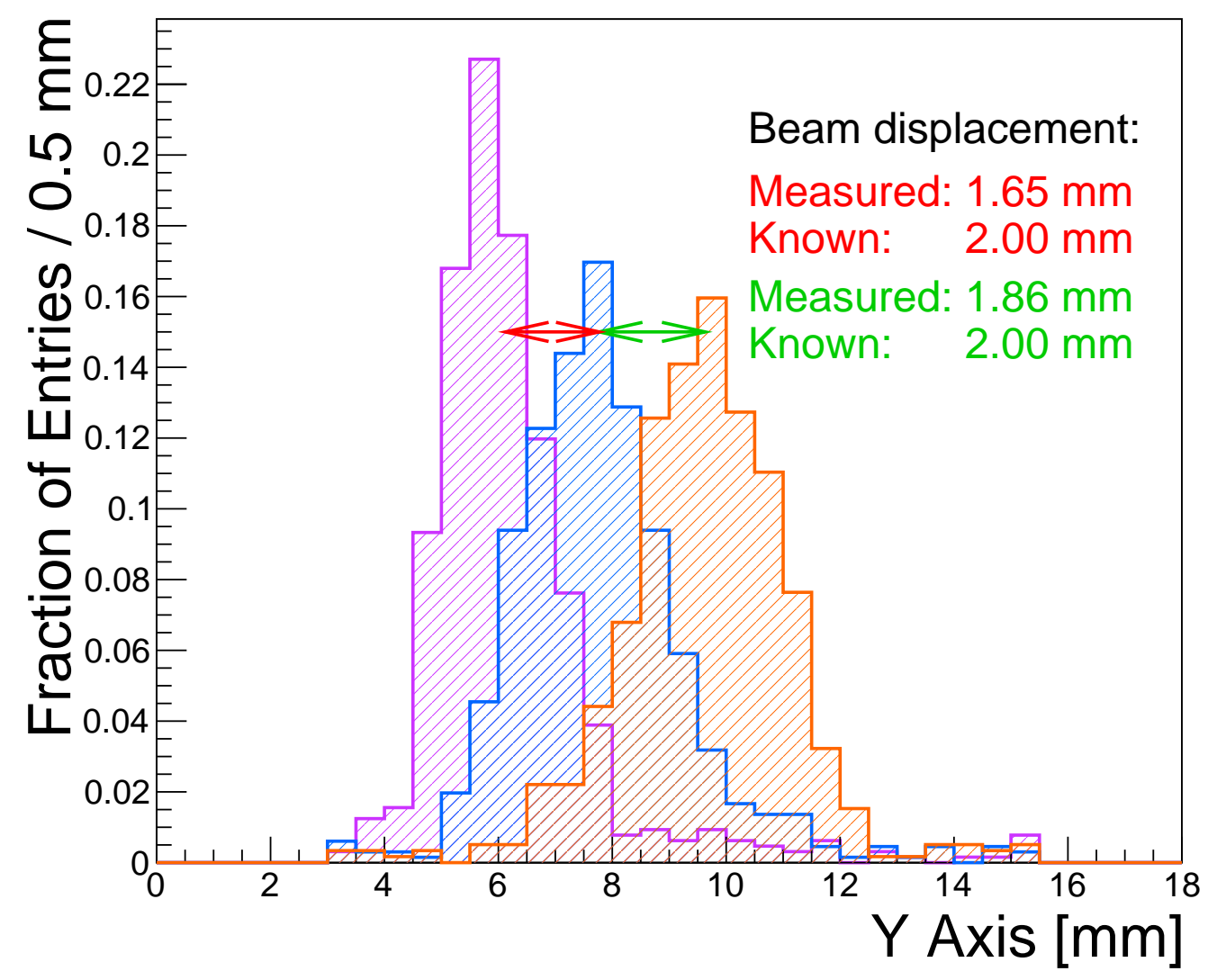

Figure 6: The distributions of reconstructed shower position in the $y$ axis is shown for the three runs corresponding to the distributions shown in Figure 5. The measured beam displacements are compared to the known displacements as recorded by the motorized stage.
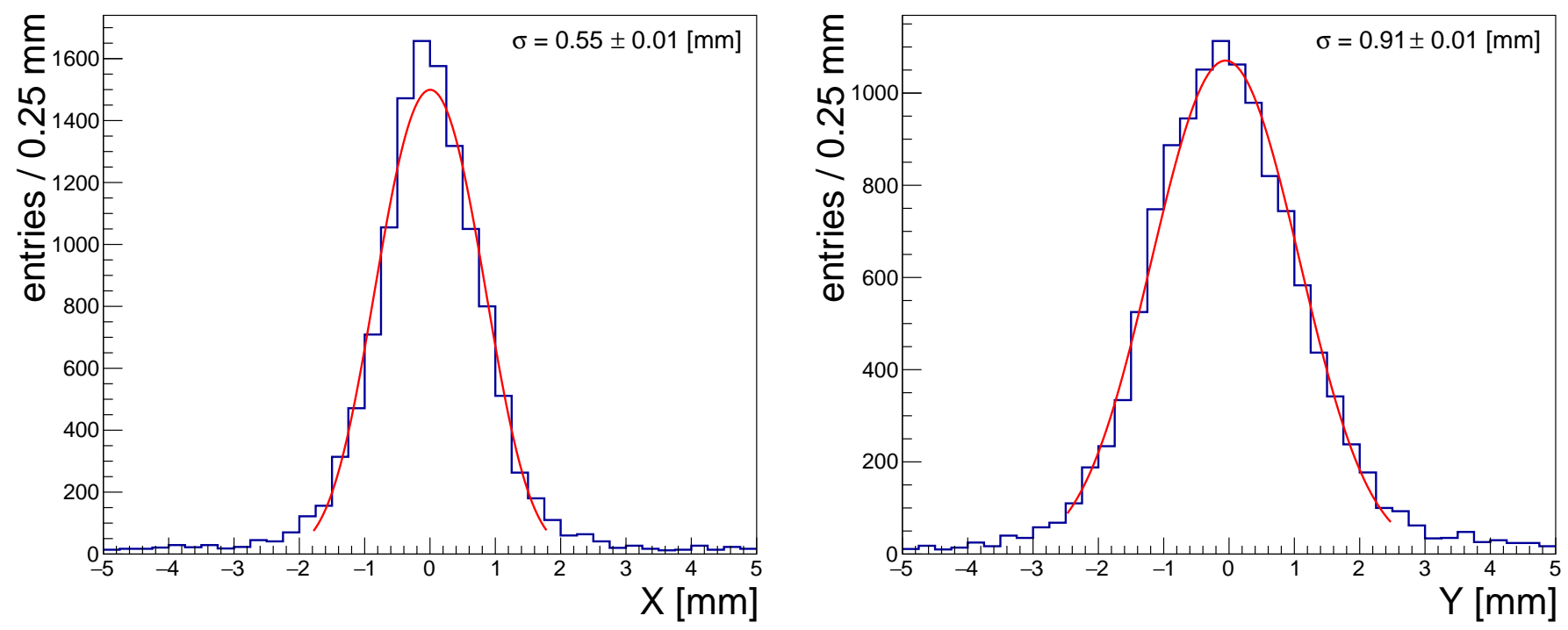

Figure 7: The distributions of the measured $x$ (left) and $y$ (right) coordinates are shown along with the fit to the resolution model. The position resolution of the EM shower as measured by the MCP-PMT detector is determined from the fit to the resolution model. 

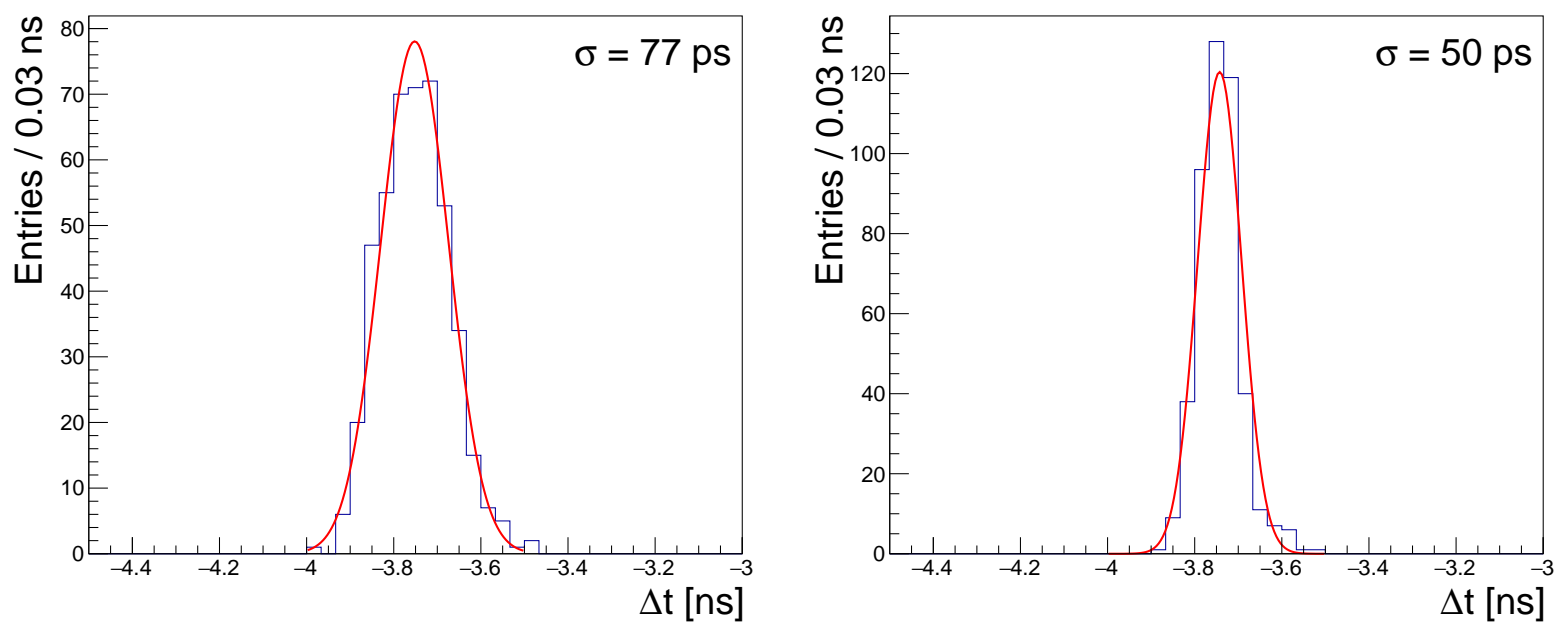

Figure 8: The time distributions obtained using the highest energy pixel (left) and the energy weighted algorithm (right) are shown for one example run. The distributions are fitted with Gaussian models, and the width parameter of the Gaussian is displayed on the plot.

around $70 \mathrm{ps}$ and $85 \mathrm{ps}$, depending on the run. Using the energy weighted algorithm improves the time resolution consistently to about 50 ps.

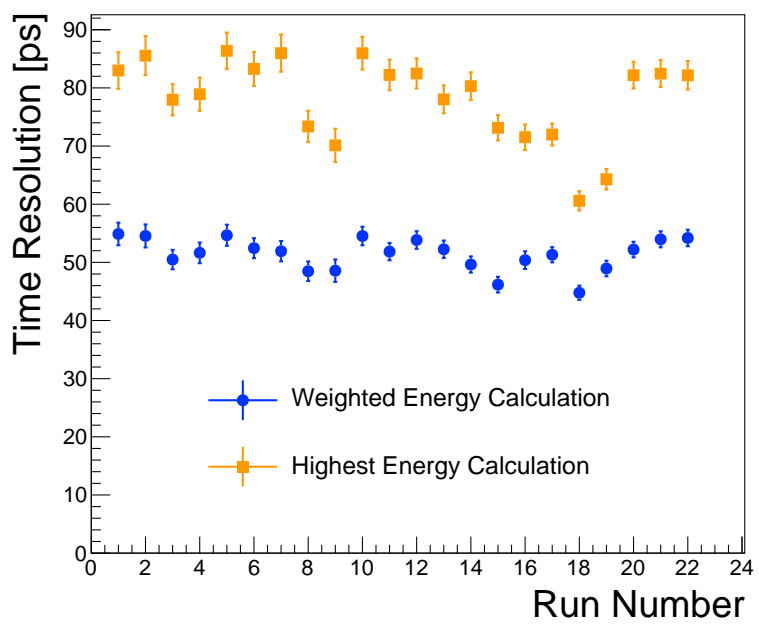

Figure 9: Time resolution found for each run. The time-stamp obtained using the energy weighting method yields time resolutions consistently below $60 \mathrm{ps}$. The time resolution measured using the single pixel with the largest signal is significantly worse.

We note that the time measurement made using the Photonis MCP-PMT typically exhibit a dependence on the pulse amplitude or integrated charge. This dependence is shown on the left of Figure 10, and is observed to be approximately the same for all pixels. We perform a correction to the time measurement based on the measured integrated charge, and we verify that the correction does flatten the dependence of the time measurement on the integrated charge as shown on the right panel of Figure 10. After performing this time measurement correction, the time resolution measurements improve to about $35 \mathrm{ps}$ and is shown in Figure 11. We performed two sets of correction procedures. In one set, labelled as "Self-Calibrated", an independent correction is derived for each run and for each pixel. In the second set, labelled as "Calibrated", a single correction is obtained for each pixel from a single run, and this correction is applied to all other runs. Figure 11 shows that this single correction is applicable to all other runs without loss of the 

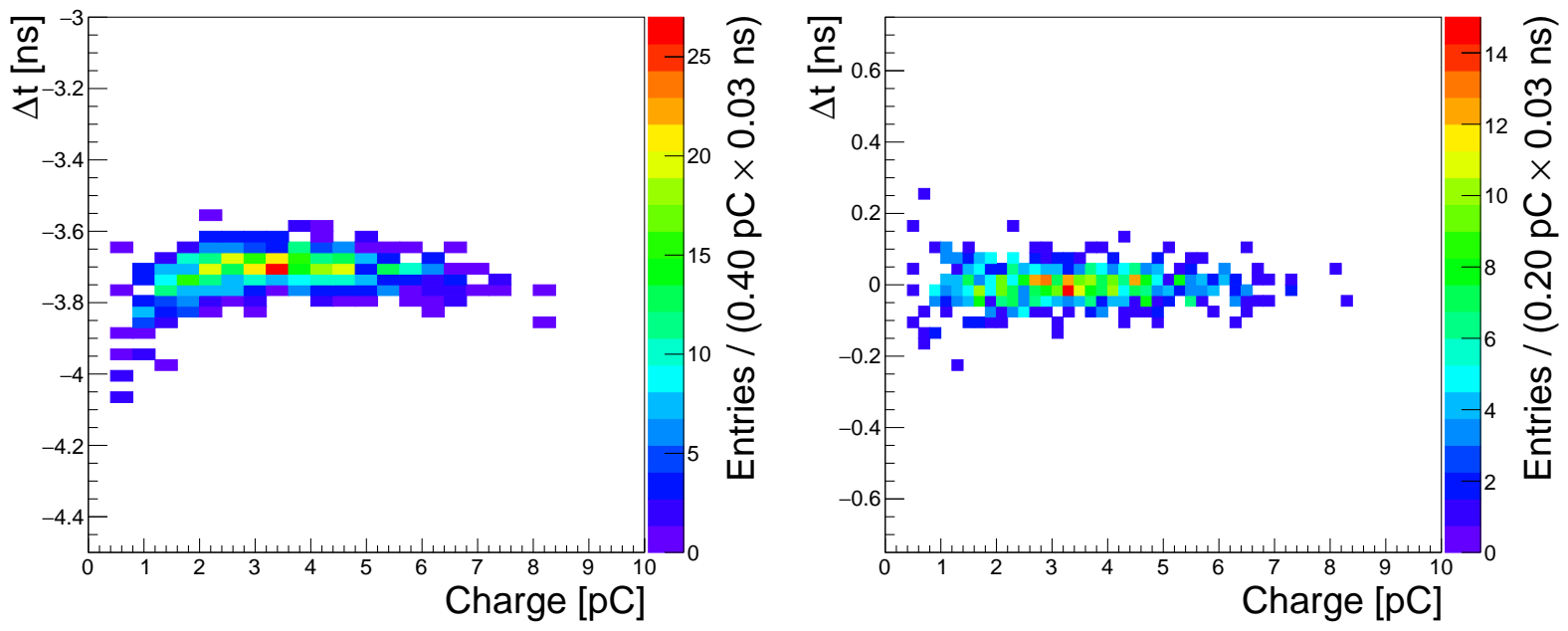

Figure 10: The correlation between the time measurement and the measured integrated charge is shown on the left for one example pixel. The same correlation after performing the time measurement correction is shown on the right.

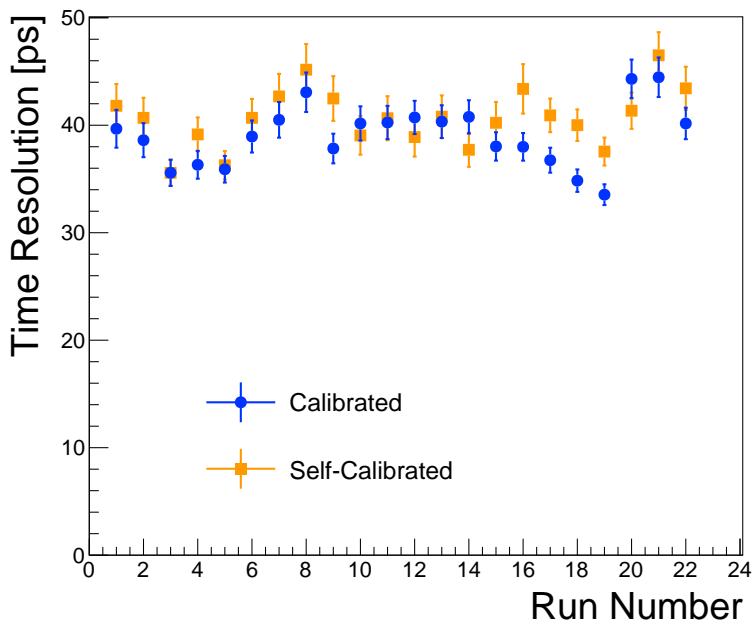

Figure 11: The time resolution of the electromagnetic shower for various runs is shown after performing the time measurement correction based on the measured integrated charge.

Finally, we study the dependence of the electromagnetic shower time resolution as a function of the number of pixels included in the energy-weighted algorithm. Figure 12 shows this dependence for one example run. We observe that the time resolution improves according to a $1 / \sqrt{N}$ scaling up to about 5-6 pixels, and then becomes flat as we include more pixels. The initial $1 / \sqrt{N}$ scaling is encouraging as it indicates that the time jitter across different pixel channels arise primarily from uncorrelated sources, and that further granularity may improve the time resolution provided that the signal is sufficiently large compared to noise. As the majority of the shower is covered by the pixels closest to the center of the shower, it is not surprising that we do not observe any improvement from the inclusion of additional pixels. 


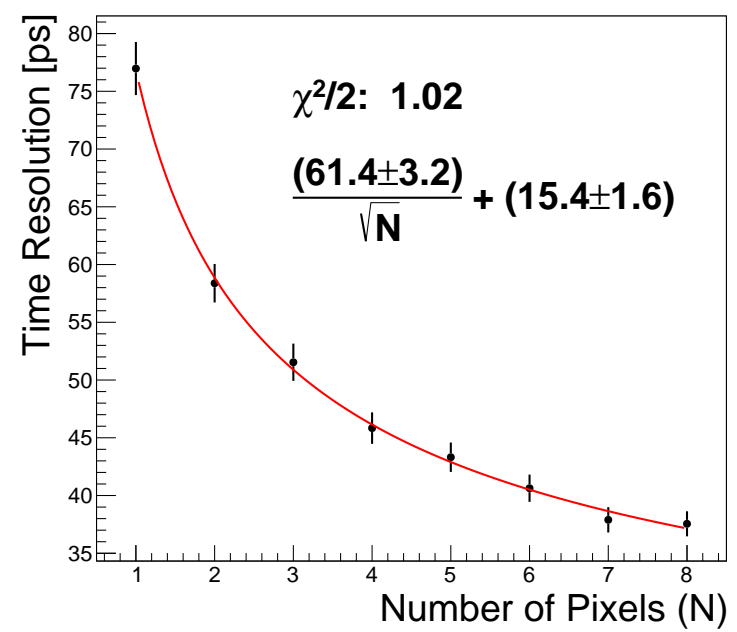

Figure 12: The time resolution is plotted as a function of the number of pixels included in the energyweighted algorithm for one example run.

\section{Summary}

We continue our studies on the development of future electromagnetic calorimeters capable of high precision energy and time measurements. Such calorimeters should provide both spatial resolution below the mm level and time resolution of $20-30 \mathrm{ps}$, in order to mitigate the detrimental effects of pileup. A highly granular readout is required to achieve these goals.

We report our results on position and time resolution measurements of a secondary emission based calorimeter prototype that used the Photonis XP85011 MCP-PMT as the active element. Using a pixelated readout of the MCP-PMT we obtain highly granular information of the shower development in the transverse plane. Combining the measurements from a $3 \times 3$-channels readout we obtain a sub-millimeter precision for position measurement, which far exceeds the $6 \mathrm{~mm}$ size of individual pixels. While the more granular readout degrades the signal to noise for each individual pixel, we demonstrate that a proper combination from independent readout channels preserves good time resolution. We show that the time resolution of the measurements improves with the increase in the number of pixels used as $1 / \sqrt{N}$, and using all pixels we achieve a time resolution of $30-40$ ps. In future measurements we intend to include larger prototypes with several layers of active material, that will allow us to explore the longitudinal development of the showers.

\section{Acknowledgements}

We would like to thank Erik Ramberg for supporting our work, and Aria Soha and the FTBF test beam facility for the good beam delivery and control. Thanks to Ewa Skup and Geoff Savage for help with operation of Cherenkov counters, and to Todd Nebel for organizing and providing supporting equipment at FTBF.

This work is supported by funding from Fermi Research Alliance, LLC under Contract No. DEAC02-07CH11359 with the United States Department of Energy, and from California Institute of Technology High Energy Physics under Contract DE-SC0011925 with the United States Department of Energy. 


\section{References}

[1] D. Anderson, A. Apresyan, A. Bornheim, J. Duarte, C. Pena, A. Ronzhin, M. Spiropulu, J. Trevor, and S. Xie, "On Timing Properties of LYSO-Based Calorimeters," Nucl. Instrum. Meth., vol. A794, pp. 7-14, 2015.

[2] A. Ronzhin, S. Los, E. Ramberg, M. Spiropulu, A. Apresyan, S. Xie, H. Kim, and A. Zatserklyaniy, "Development of a new fast shower maximum detector based on microchannel plates photomultipliers (mcp-pmt) as an active element," Nucl. Instrum. Meth., vol. A759, pp. 65 $73,2014$.

[3] A. Ronzhin, S. Los, E. Ramberg, A. Apresyan, S. Xie, M. Spiropulu, and H. Kim, "Direct tests of micro channel plates as the active element of a new shower maximum detector," Nucl. Instrum. Meth., vol. A795, pp. 52-57, 2015.

[4] A. Ronzhin, S. Los, E. Ramberg, A. Apresyan, S. Xie, M. Spiropulu, and H. Kim, "Study of the timing performance of micro-channel plate photomultiplier for use as an active layer in a shower maximum detector," Nucl. Instrum. Meth., vol. A795, pp. 288 - 292, 2015.

[5] A. A. Derevshchikov, V. Yu. Khodyrev, V. I. Kryshkin, V. E. Rakhmatov, and A. I. Ronzhin, "On possibility to make a new type of calorimeter: Radiation resistant and fast," Tech. Rep. IFVE-90-99, IHEP, Protvino, Russia, 1990.

[6] A. Albayrak-Yetkin et al., "Secondary Emission Calorimetry: Fast and Radiation-Hard," in Community Summer Study 2013: Snowmass on the Mississippi (CSS2013) Minneapolis, MN, USA, July 29-August 6, 2013, 2013.

[7] L. Brianza, F. Cavallari, D. D. Re, S. Gelli, A. Ghezzi, C. Gotti, P. Govoni, C. J. Lopez, A. Martelli, B. Marzocchi, P. Meridiani, G. Organtini, R. Paramatti, L. Perni, S. Pigazzini, S. Rahatlou, C. Rovelli, F. Santanastasio, T. T. de Fatis, and N. Trevisani, "Response of microchannel plates to single particles and to electromagnetic showers," Nucl. Instrum. Meth., vol. A797, pp. 216 - 221, 2015.

[8] D. Grondin and D. Jeans, "Next Generation CALICE Electromagnetic Calorimeter," in International Linear Collider Workshop 2010 (LCWS10 \& ILC10) Beijing, China, March 26-30, 2010, 2010.

[9] J. Butler, D. Contardo, M. Klute, J. Mans, and L. Silvestris, "Technical Proposal for the PhaseII Upgrade of the CMS Detector," Tech. Rep. CERN-LHCC-2015-010. LHCC-P-008, CERN, Geneva. Geneva, Jun 2015. 